\title{
ASO Author Reflections: Locoregional Therapy in De Novo Bone-Only Metastatic Breast Cancer Provides Survival Advantage Regardless of Therapy Sequencing
}

\author{
Atilla Soran, MD, MPH, FNCBC, FACS ${ }^{1}$, Lutfi Dogan, $\mathrm{MD}^{2}$, and Serdar Ozbas, $\mathrm{MD}^{3}$ \\ ${ }^{1}$ Department of Surgical Oncology, University of Pittsburgh Medical Center, Magee-Womens Hospital, Pittsburgh; \\ ${ }^{2}$ Department of Surgical Oncology, University of Health Sciences, Ankara Oncology Training and Research Hospital, \\ Ankara, Turkey; ${ }^{3}$ Private, Breast and Endocrine Surgeon, Ankara, Turkey
}

\section{PAST}

It is known that locoregional therapy (LRT) in metastatic breast cancer contributes to local control and also prolongs systemic progression-free survival and overall survival. ${ }^{1}$ However, metastatic breast cancer reflects a heterogeneous group of diseases with a broad spectrum. Tumor load, metastatic site, and biological characteristics of the tumor determine the clinical course. Although having a metastatic disease is the most significant factor in shortening overall survival, bone-only metastasis is a special entity in terms of both its biological behavior and clinical course. ${ }^{2}$ Bone-only metastases are present in more than half of de novo metastatic patients. ${ }^{3}$ Retrospective trials concluded that LRT should be evaluated in bone-only metastatic patients in prospective studies and that the extent, type, and sequencing of LRT should be studied.

\section{PRESENT}

Protocol BOMET MF14-01 is a multicenter prospective registry study. Patients with bone-only metastases at diagnosis were included. The study had three groups of patients: group 1 received only systemic therapy, group 2 systemic therapy prior to LRT, and group 3 received LRT after systemic therapy. In this study, LRT prolonged overall survival and systemic progression-free survival

(C) Society of Surgical Oncology 2021

First Received: 18 January 2021

Accepted: 19 January 2021;

Published Online: 5 February 2021

L. Dogan, MD

e-mail: lutfidogan1@yahoo.com with reducing locoregional recurrence. ${ }^{4}$ The timing of LRT was not an independent contributing factor on survival. Although survival was longer in patients with solitary and oligometastasis, overall survival was longer in multiple metastatic patients who had LRT as well.

\section{FUTURE}

Such a large prospective multicenter registry study focusing on a single metastatic site has not been presented before. For current practice, while planning treatment of patients with only bone metastases at the time of diagnosis, the primary LRT alternative can be discussed in tumor board and with patient. To adapt surgery in guidelines and clinical practice routinely, there is an unmet need for randomized studies to evaluate of LRT in patients with bone-only metastasis.

DISCLOSURES The authors declare that they have no conflict of interest.

\section{REFERENCES}

1. Soran A, Ozmen V, Ozbas S, et al. randomized trial comparing resection of primary tumor with no surgery in stage IV breast cancer at presentation: protocol MF07-01. Ann SurgOncol. 2018;25:3141-9.

2. Coleman RE. Clinical features of metastatic bone disease and risk of skeletal morbidity. Clin Cancer Res. 2006;12:6243-9.

3. Noone AMHN, Krapcho M, Miller D, et al. editors. SEER cancer statistics review, 1975-2015. J Natl Cancer Inst. 2018.

4. Soran A, Dogan L, Isik A, et al. The effect of primary surgery in patients with stage IV breast cancer with bone metastasis only (protocol BOMET MF 14-01); a multi-center, registry study. Ann Surg Oncol. 2021. https://doi.org/10.1245/s10434-021-09621-8

Publisher's Note Springer Nature remains neutral with regard to jurisdictional claims in published maps and institutional affiliations. 